\title{
Phylogeographical pattern of Francisella tularensis in a nationwide outbreak of tularaemia in Norway, 2011
}

J E Afset ${ }^{1,2}$, K W Larssen², K Bergh ${ }^{1,2}$, A Lärkeryd³, A Sjödin³, A Johansson4, M Forsman (mats.forsman@foi.se) ${ }^{3}$

1. Department of Laboratory Medicine, Children's and Women's Health, Faculty of Medicine, Norwegian University of Science and Technology, Trondheim, Norway

2. Department of Medical Microbiology, St. Olavs Hospital, University Hospital, Trondheim, Norway

3. Division of CBRN Security and Defence, FOI - Swedish Defence Research Agency, Umeå, Sweden

4. Department of Clinical Microbiology, The Laboratory for Molecular Infection Medicine Sweden (MIMS), Umeå University, Umeå, Sweden

In 2011, a nationwide outbreak of tularaemia occurred in Norway with 180 recorded cases. It was associated with the largest peak in lemming density seen in 40 years. Francisella tularensis was isolated from 18 patients. To study the geographical distribution of $F$. tularensis genotypes in Norway and correlate genotype with epidemiology and clinical presentation, we performed whole genome sequencing of patient isolates. All 18 genomes from the outbreak carried genetic signatures of $F$. tularensis subsp. holarctica and were assigned to genetic clades using canonical single nucleotide polymorphisms. Ten isolates were assigned to major genetic clade B.6 (subclade B.7), seven to clade B.12, and one to clade B.4. The B.6 subclade B.7 was most common in southern and central Norway, while clade B.12 was evenly distributed between the southern, central and northern parts of the country. There was no association between genotype and clinical presentation of tularaemia, time of year or specimen type. We found extensive sequence similarity with $F$. tularensis subsp. holarctica genomes from high-endemic tularaemia areas in Sweden. Finding nearly identical genomes across large geographical distances in Norway and Sweden imply a life cycle of the bacterium without replication between the outbreaks and raise new questions about long-range migration mechanisms.

\section{Introduction}

Tularaemia is a zoonotic infection caused by the bacterium Francisella tularensis, a pathogen with extreme infectivity and a potential biothreat [1]. Several subspecies have been recognised, of which $F$. tularensis subsp. holarctica (or type B) is present in Europe. Tularaemia is a rare disease in a global context, making the experience with outbreak investigations very limited. The Scandinavian countries, however, quite regularly experience outbreaks in humans involving tens to hundreds of patients. In Norway, several outbreaks have been attributed to $F$. tularensis contamination of water wells by dead rodents. The exposure to infection by drinking water results in an oropharyngeal clinical form of tularaemia in humans and this is the most common form reported in Norway [2-4]. For unknown reasons, the incidence rate of tularaemia in humans is lower in Norway than in the two other Nordic countries Finland and Sweden. While a mean annual number of 34 (range: 11-66) tularaemia cases were reported in Norway 2006 to 2010, Finland and Sweden reported during the same period a mean annual number of 298 and 305, respectively [5]. The mode of transmission to humans is also different because tularaemia in Finland and Sweden is generally transmitted by the bite of an arthropod taking a blood meal resulting in the ulceroglandular form of tularaemia $[6,7]$. The reasons for the epidemiological differences in incidence and clinical form of tularaemia between the three countries are unknown.

Distinct genetic subpopulations (major phylogenetic clades) have been identified among $F$. tularensis subsp. holarctica strains [8]. High-resolution molecular methods including whole genome sequencing distinguish four major genetic clades denoted B.12, B.6, B.4 and B.16 [9-11]. The clades occur with different frequency in different geographical areas [12-15]. Recent studies in Sweden have indicated phylogeographical patterns both in local outbreaks and across larger geographical distances. These studies from Sweden also identified areas where $F$. tularensis persisted over several years and spatial associations of certain genetic subpopulations [16]. Little is known about phylogeographical patterns of $F$. tularensis in Norway.

In 2011, a large outbreak of tularaemia occurred in Norway with a total of 180 cases coinciding with the highest density of lemmings recorded in the last forty years [17]. Although the high incidence lasted 
Genetic clade and subclade of human Francisella tularensis subsp. holarctica isolates in relation to type of specimen, clinical classification, time of infection and geographical distribution, Norway, $2011(\mathrm{n}=18)$

\begin{tabular}{|c|c|c|c|c|c|c|}
\hline Strain ID & Type of specimen & Clinical classification & Time of year & $\begin{array}{l}\text { Geographical } \\
\text { region }\end{array}$ & $\begin{array}{l}\text { Genetic clade/ } \\
\text { subclade }\end{array}$ & $\begin{array}{c}\text { NCBI accession } \\
\text { number }\end{array}$ \\
\hline $\mathrm{NO}-1 / 2011$ & Blood & Typhoidal & January & Central & B.7 & JPPD000000000 \\
\hline $\mathrm{NO}-2 / 2011$ & Blood & Respiratory & February & Central & B.12 & JPMM00000000 \\
\hline $\mathrm{NO}-3 / 2011$ & Blood & Respiratory & February & South & B.7 & JPPE00000000 \\
\hline $\mathrm{NO}-4 / 2011$ & Blood & Typhoidal & March & Central & B.7 & JPMJ00000000 \\
\hline NO-5/2011 & Aspirate & Ulceroglandular & May & South & B.12 & JPPFo0000000 \\
\hline NO-6/2011 & Exudate from ulcer & Ulceroglandular & July & South & B.7 & JPMK000000000 \\
\hline NO-7/2011 & Exudate from ulcer & Ulceroglandular & August & North & B.12 & JPMLO00000000 \\
\hline NO-8/2011 & Exudate from ulcer & Ulceroglandular & August & South & B.12 & JPPG00000000 \\
\hline NO-9/2011 & Exudate from ulcer & Ulceroglandular & August & South & B.7 & JPPI00000000 \\
\hline NO-10/2011 & Tissue biopsy & Ulceroglandular & August & South & B.7 & JPPHOO000000 \\
\hline NO-11/2011 & Tissue biopsy & Respiratory & August & South & B.7 & JPPJ00000000 \\
\hline NO-12/2011 & Exudate from ulcer & Ulceroglandular & August & South & B.12 & JPMNO0000000 \\
\hline NO-13/2011 & Aspirate & Ulceroglandular & August & South & B.7 & JPMO00000000 \\
\hline NO-14/2011 & Blood & Typhoidal & September & North & B.7 & JPPK00000000 \\
\hline NO-15/2011 & Blood & Respiratory & November & North & B. 4 & JPMP00000000 \\
\hline NO-16/2011 & Exudate from ulcer & Ulceroglandular & November & Central & B.7 & JPMQ00000000 \\
\hline NO-17/2011 & Exudate from ulcer & Ulceroglandular & October & North & B.12 & JPMROo0000000 \\
\hline NO-18/2011 & Blood & Glandular & November & North & B.12 & JPMSO00000000 \\
\hline
\end{tabular}

NCBI: National Center for Biotechnology Information.

throughout the year, there were clear differences in epidemiology between seasons, both in the incidences in different geographical areas and in the clinical forms of tularaemia recorded. The outbreak started in January 2011 in central Norway (Sør-Trøndelag and neighbouring counties) with mainly cases of oropharyngeal tularaemia linked to the use of drinking water from private wells [18]. This part of the outbreak lasted until April. In the period from May to September, sporadic cases occurred scattered throughout the country with increasing frequency of the ulceroglandular form of tularaemia linked to insect bites [17]. From October to December, many cases of tularaemia were reported from the north of Norway, equally distributed between the oropharyngeal, glandular, typhoidal and respiratory forms of tularaemia. For comparison, the number of tularaemia cases reported in 2011 in Sweden was 349 , slightly above the annual average of the period 2006 to $2010[5,19]$.

The aim of this project was to use whole genome sequencing for genotyping of $F$. tularensis cultured from human specimens during the outbreak in 2011 to investigate the genotype distribution of tularaemia in Norway. We also wanted to analyse associations of genotype with epidemiological characteristics and disease presentation and identify patterns of spread of the bacterium.

\section{Methods}

\section{Isolation of clinical strains}

F. tularensis was cultured from 18 of the 180 patients diagnosed with tularaemia in Norway in 2011. All these isolates were included in the study. F. tularensis was cultured from four of 57 tularaemia cases during the period January to April, from 10 of 40 cases in the period May to September (seven of these in August), and from four of 83 cases in the period October to December. The isolates were from blood $(n=7)$, skin ulcer $(n=7)$, tissue biopsy $(n=2)$ and aspirate $(n=2)$ specimens from patients living in nine different counties (Table).

The study, which was based on informed written consent from each patient, was approved by the Regional Committee for Medical and Health Research Ethics, central Norway (project 2012/867).

\section{Genome sequencing and assembly}

Genome sequencing was performed using $100 \mathrm{bp}$ paired-end libraries on an Illumina HiSeq 2000 machine (Illumina, San Diego, CA). The reads were assembled de novo using the ABySS [20]. The raw sequence reads were mapped back against the draft genome using Bowtie 2 [21] allowing identification of uncertain positions in the genome with VarScan 2 [22]. Positions reported having a second variant with frequency higher than $20 \%$ were subsequently masked. The overall genome coverage was ca 850 -fold. 


\section{FIGURE 1}

Neighbour-joining phylogenetic tree based on whole genome alignment of Francisella tularensis sequences $(\mathrm{n}=53)$

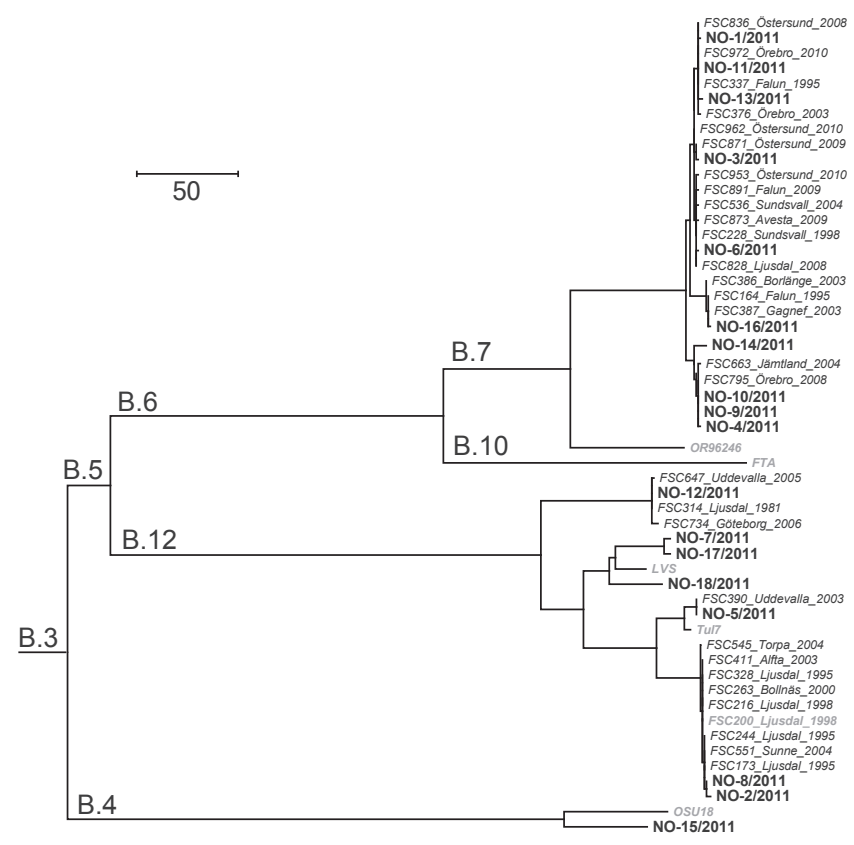

The tree is based on alignment of $1,613,828$ shared nucleotide positions and rooted using FSC022, a B.16 strain (not shown), and drawn to scale with branch length representing the number of nucleotide changes.

Bold: Norwegian isolates; italics: Swedish strains within a distance of two nucleotide polymorphisms from any Norwegian strain; grey: reference strains.

\section{In silico screening of Francisella strains}

Genome sequences were initially in silico screened by the CanSNPer software [23] using published canonical SNP Francisella tularensis subsp. holarctica markers [9-13]. To select a suitable set of reference strains, the $18 \mathrm{draft}$ genomes were aligned with Swedish F. tularensis subsp. holarctica genomes from the same genetic clades, as determined by CanSNPer $(n=313)$. Using this alignment, reference strains that were within a distance of two single nucleotide polymorphisms (SNPs) from any Norwegian sequence were selected for further analysis.

\section{Genome alignment and phylogenetic tree construction}

A multiple genome alignment of 53 strains was generated by concatenation of a number of pairwise alignments where each strain was aligned against the reference strain $\mathrm{FSC}_{200}$ [24] using progressive MAUVE [25]. In addition to the 18 Norwegian and 29 selected Swedish strains, six common reference genomes were included (OR96246, FTNFoo2-0o [26], LVS, Tul7 [13], $\mathrm{FSC}_{200}$ [24], OSU18 [27], and the tree was rooted in FSC022 [28]. The phylogenetic tree was constructed in MEGA6 [29].

\section{Results}

All 18 isolates of $F$. tularensis cultured from patients in Norway in 2011 were identified as $F$. tularensis subsp. holarctica based on whole genome sequence genotyping. The distribution of the isolates in genetic clades and subclades is shown in the Table and Figure 1. Seven of the isolates were assigned to the major genetic clade B.12, one to clade B.4 and 10 isolates to a subclade denoted B.7 belonging in the major clade B.6.

\section{Type of specimen}

Among the seven isolates cultured from blood, four belonged to subclade B.7, while two belonged to B.12 and one to clade B.4 (Table). Four isolates from skin ulcers belonged to subclade B.7 and three to B.12. The two isolates from aspirates belonged to B.12 and B.7, respectively, while both isolates from tissue biopsies belonged to subclade B.7.

\section{Clinical form of tularaemia}

Ten of the $F$. tularensis isolates were from cases of ulceroglandular tularaemia (Table). Five of these isolates belonged to subclade B.7; the other five strains belonged to clade B.12. Two of the four isolates from patients with a respiratory form of tularaemia belonged to subclade B.7, while the other two belonged to B.12 and B.4. The three isolates recovered from patients with typhoidal tularaemia all belonged to subclade B.7. Finally, the isolate from a patient with glandular tularaemia was of clade B.12.

\section{Mode of infection}

Three of the six $F$. tularensis isolates cultured from ulcers caused by insect bite belonged to subclade B.7 while the three others belonged to subclade B.12. The three isolates recovered from patients with typhoidal tularaemia who reported to have drunk water from a private well, belonged to subclade B.7. Inhalation (clade B.12), outdoor work in a farm (subclade B.7), handling of a dead rabbit (subclade B.7), stab wound while handling fish (clade B.12), and hunting and other outdoors activities (clade B.12) were reported as likely mode of infection for five other isolates. For the four remaining isolates, information on the likely mode of infection was not available.

\section{Seasonal distribution}

Three of the four $F$. tularensis isolates cultured in the period January to April belonged to subclade B.7 and one belonged to clade B.12 (Table). In the period May to September, 10 cases were culture-positive, eight of whom had the ulceroglandular form of tularaemia. Six of the isolates from this period belonged to subclade B7, and four to clade B.12. Among the four isolates recovered during the period October to December, two belonged to subclade B.12, one to clade B.4 and one to subclade B.7.

\section{Geographical distribution}

Among the nine isolates recovered from patients in southern Norway, six belonged to subclade B.7 and 


\section{FIGURE 2}

Geographical distribution of near-identical (within a distance of two single nucleotide polymorphisms across the genome) Francisella tularensis strains in Norway $(\mathrm{n}=18)$ and Sweden $(\mathrm{n}=29)$

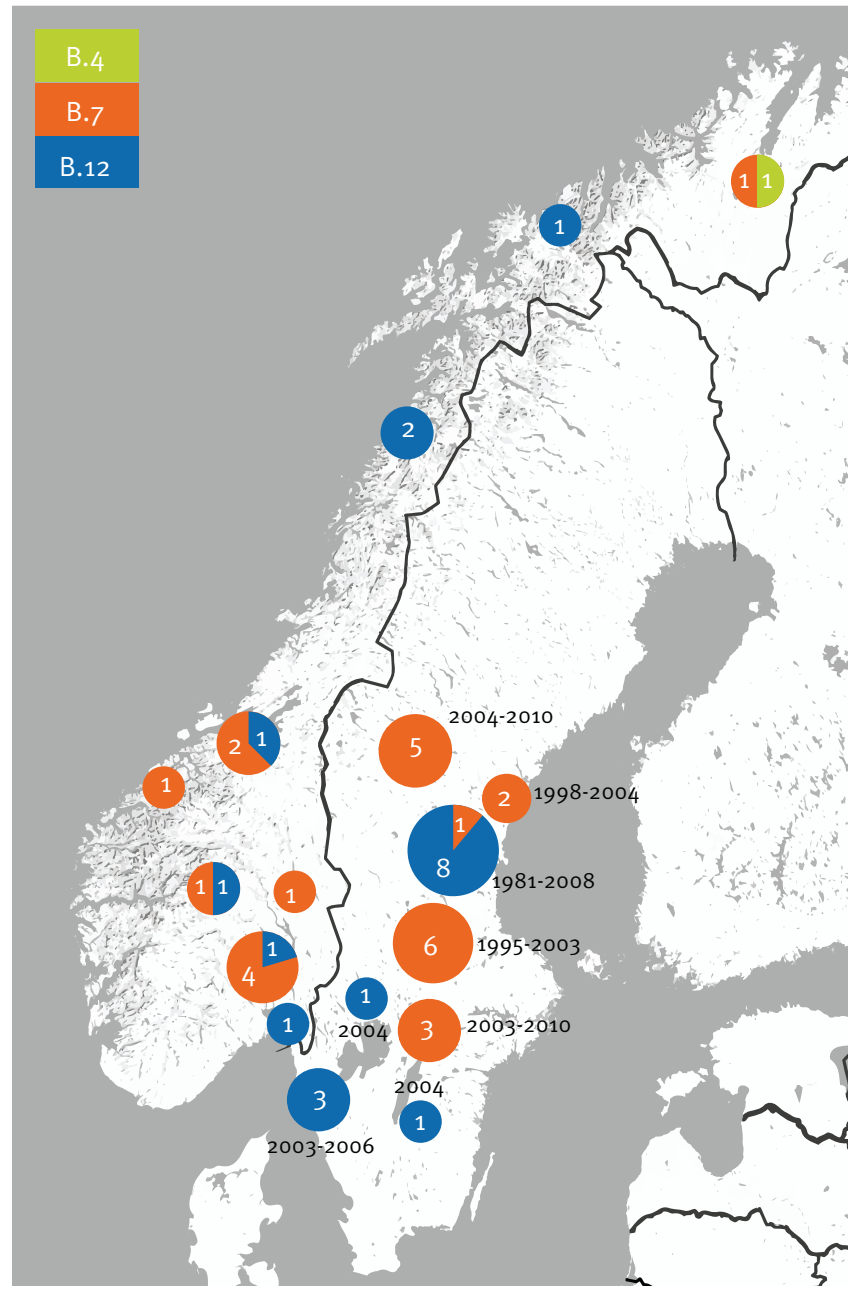

Strains included on the map are the same as those listed in the different clades and subclades (B.7, B.12 and B.4) in the Neighbour-joining tree in Figure 1.

three to clade B.12 (Figure 2). In comparison, three of the four isolates from central Norway belonged to subclade B.7 and one belonged to clade B.12, while one of the five isolates from patients living in northern Norway belonged to subclade B.7, three belonged to B.12 and one to clade B.4. Two patients living in southern Norway provided information that they may have contracted the infection while walking in the mountains or working on a farm without specifying where. For a third patient, information on the place of infection was not available. One patient living in northern Norway reported infection after an insect bite while travelling in Sweden (strain NO-7/2011).

\section{Comparison with Swedish strains}

Among the 313 archived Swedish F. tularensis strains included in the analysis, the genomes of 29 strains differed at two or fewer SNPs compared with the outbreak genomes from Norway (Figure 1). All those 29 strains had been isolated during the period 1981 to 2010 in areas highly endemic for tularaemia in central Sweden (Figure 2). Since culture for $F$. tularensis was not routinely performed in Norwegian medical microbiology laboratories before 2011, archived genome sequences from Norwegian $F$. tularensis isolates from that period were not available for this study.

\section{Discussion}

In this study we have shown that 10 of $18 \mathrm{~F}$. tularensis subsp. holarctica isolates from human tularaemia cases from the outbreak in Norway in 2011 belonged to the genetic subclade B.7, while seven isolates belonged to clade B.12 and one to clade B.4. Isolates from the clade B.12 showed higher genomic variability than those belonging to the subclade B.7. Comparison of the isolates from the outbreak in Norway with archived genome sequences from Sweden since 1981 revealed strong sequence identity between some Norwegian and Swedish isolates from high-endemic areas. Twenty-nine isolates from Sweden differed at two or fewer SNPs from one or several isolates from Norway (Figure 1). No information is available on the mutation rate for $F$. tularensis in general or during replication in hosts. Thus, the meaning of near-identical strains (a maximum of two SNPs across the genome) cannot be put into context of mutation rate. Because both countries are located on the Scandinavian Peninsula and share a long border, our results support a common ecology of $F$. tularensis on the Peninsula, although the incidence and mode of transmission of tularaemia differ between Norway and Sweden. Reasons for differences in the epidemiology of tularaemia between the two countries are not known, but one possible explanation of the higher proportion of oropharyngeal cases in Norway could be that private wells for drinking water might be more common in Norway [18]. Our data suggest that several of the genetic clades have moved long geographical distances and that near-identical genetic clones of the bacterium were found to be far apart both geographically and temporally. An alternative explanation may be that identical genotypes of $F$. tularensis have evolved by parallel evolution in many geographical regions of the Scandinavian Peninsula. Our results may be consistent with a life cycle of the bacterium that includes a state of quiescence (absence or very low level of replication) for long periods of time between outbreaks [30,31].

Both subclade B.7 and clade B.12 of F. tularensis subsp. holarctica were detected in tularaemia cases from all three regions of Norway. However, while subclade B.7 were mostly found in the southern and central regions of the country, the clade B.12 isolates were more evenly distributed between the regions (Figure 2).

Before the outbreak in 2011 and since the disease became a notifiable disease in Norway in 1977, only five cases of human tularaemia have been reported from the northernmost county Finnmark. Altogether 62 cases of tularaemia were reported from this county 
during this outbreak which in Finnmark lasted until 2012. Considering the high sequence identity between the B.7 isolate from Finnmark (strain NO-14/2011) and other B.7 isolates both from southern Norway and central Sweden, the emergence of tularaemia in Finnmark was most probably caused by bacteria already present in the environment. Their activation and amplification may have required additional factors such as the large outbreak in lemmings that occurred in Finnmark in 2011. This raises questions about what mechanisms allowed these near-identical strains to spread across such large geographical distances. Birds have been implicated in the transportation of $F$. tularensis $[32,33]$. However, while occasional transportation by birds cannot be excluded, the general geographical pattern of near-identical strains found in this study is not characteristic of the north-south migration routes of migratory birds on the Scandinavian Peninsula. Many different mechanisms for dissemination could be envisaged, such as long-range aerosol transport by wind, carriage by arthropod vectors and/or infected migratory wild animals, or a combination of several mechanisms. Further research into this phenomenon is needed.

In this study we found no statistical association between the genotype of $F$. tularensis and type of specimen, clinical presentation, mode of transmission or time of the year when the specimen was collected, although the low number of bacterial strains may have obscured weak associations (Table). Our findings are in analogy with those reported in a recent genomic study of a respiratory tularaemia outbreak in Sweden [30]. In the latter study it was shown that the respiratory form of tularaemia was not tied to specific genotypes of $F$. tularensis and that outbreak genomes shared high sequence similarity with archived isolates originating from patients from distant geographical regions and collected up to 10 years apart [30]. Despite the mentioned lack of significant association in our study, it is worth noting that $F$. tularensis of clade B.12 was found mainly in patients with ulceroglandular tularaemia, and that all three isolates from patients with typhoidal tularaemia belonged to subclade B.7 (Table).

We were able to culture $F$. tularensis from only $10 \%$ of the tularaemia cases in 2011. Few reports are available on the sensitivity of culture in the diagnosis of tularaemia, but a low sensitivity has been reported in an outbreak of orpharyngeal tularaemia [34]. This, as well as the overrepresentation of the ulceroglandular form of the disease among culture-positive cases, make it difficult to assess the representativeness of the isolates for the whole outbreak. Another limitation is that for three of the isolates, we did not have data on where the patients had been infected.

In conclusion, 18 isolates of $F$. tularensis subsp. holarctica from a nationwide outbreak of tularaemia in Norway were genotyped by whole genome sequencing: among those, subclade B.7 was most frequent (10 isolates), followed by clade B.12 (seven isolates) and B.4 (one isolate). We found no association between genotype and clinical presentation of tularaemia, time of year of disease, or specimen type. Subclade B.7 was most common in southern and central Norway, while three of the five isolates from patients from northern Norway belonged to clade B.12. The isolates from this study showed near-identity with archived genomes from high-endemic areas in Sweden.

\section{Acknowledgments}

We thank Norwegian microbiology laboratories where $F$. tularensis were isolated from patient samples for submitting the isolates to the national reference laboratory, and laboratory technicians at Department of Medical Microbiology, St Olavs Hospital for technical support. Sequencing was mainly performed by the SNP\&SEQ Technology Platform which is supported by Uppsala University, Uppsala University Hospital, Science for Life Laboratory in Uppsala, Sweden and the Swedish Research Council. This project was supported in part by the Swedish Ministry of Defence (No. A404014).

\section{Conflict of interest}

None declared.

\section{Authors' contributions}

All of the authors collaborated in the presented work. JEA, $M F, A S, A L, A J$ defined the research theme. KWL, JEA and KB did the primary characterisation of the bacterial strains, applied for ethical approval of the study and collected patient data. JEA, MF, AS AL analysed data, interpreted results and wrote the draft manuscript. All authors have contributed to and approved the manuscript.

References

1. World Health Organization (WHO). WHO guidelines on tularaemia: epidemic and pandemic alert and response. Geneva: WHO; 2007. Available from: http://www.who.int/csr/ resources/publications/WHO_CDS_EPR_2007_7.pdf

2. Brantsaeter $A B$, Krogh T, Radtke A, Nygard K. Tularaemia outbreak in northern Norway. Euro Surveill. 2007;12(3):E070329.2. PMID:17439796

3. Rike HF, Vigerust A, Bergh K. Vannbårent utbrudd av tularemia (harepest) i Midtre Gauldal. [A waterborne outbreak of tularaemia in Midtre-Gauldal]. Oslo: Norwegian Institute of Public Health; 2003. Norwegian.

4. Melien P, Holsdal R. Tularemi i Meldal - en vanskelig diagnose? [Tularaemia in Meldal- a difficult diagnosis?]. Oslo: Norwegian Institute of Public Health; 2008. Norwegian. Available from: http://www.fhi.no/dav/dzeebf5efa.pdf

5. European Center for Disease Prevention and Control (ECDC). Annual epidemiological report. Reporting on 2010 surveillance data and 2011 epidemic intelligence data. Stockholm:

ECDC. Mar 2013. Available from: http://www.ecdc.europa. eu/en/publications/Publications/Annual-EpidemiologicalReport-2012.pdf

6. Eliasson H, Lindbäck J, Nuorti JP, Arneborn M, Giesecke J, Tegnell A. The 2000 tularemia outbreak: a case-control study of risk factors in disease-endemic and emergent areas, Sweden. Emerg Infect Dis. 2002;8(9):956-60. http://dx.doi. org/10.3201/eido809.020051 PMID:12194773

7. Rossow H, Ollgren J, Klemets P, Pietarinen I, Saikku J. Pekkanen E, et al. Risk factors for pneumonic and ulceroglandular tularaemia in Finland: a population-based case-control study. Epidemiol Infect. 2014;142(10):220716. http://dx.doi.org/10.1017/S0950268813002999 PMID:24289963

8. Johansson A, Farlow J, Larsson P, Dukerich M, Chambers E, Byström M, et al. Worldwide genetic relationships among Francisella tularensis isolates determined by 
multiple-locus variable-number tandem repeat analysis. J Bacteriol. 2004;186(17):5808-18. http://dx.doi.org/10.1128/ JB.186.17.5808-5818.2004 PMID:15317786

9. Vogler AJ, Birdsell D, Price LB, Bowers JR, Beckstrom-Sternberg SM, Auerbach RK, et al. Phylogeography of Francisella tularensis: global expansion of a highly fit clone. J Bacteriol. 2009;191(8):2474-84. http://dx.doi.org/10.1128/JB.01786-08 PMID:19251856

10. Karlsson E, Svensson K, Lindgren P, Byström M, Sjödin A, Forsman M, et al. The phylogeographic pattern of Francisella tularensis in Sweden indicates a Scandinavian origin of Eurosiberian tularaemia. Environ Microbiol. 2013;15(2):634-45. http://dx.doi.org/10.1111/1462-2920.12052 PMID:23253075

11. Svensson K, Granberg M, Karlsson L, Neubauerova V, Forsman M, Johansson A. A real-time PCR array for hierarchical identification of Francisella isolates. PLoS ONE. 2009;4(12):e8360. http://dx.doi.org/10.1371/journal. pone.0008360 PMID:20027310

12. Chanturia G, Birdsell DN, Kekelidze M, Zhgenti E, Babuadze G, Tsertsvadze N, et al. Phylogeography of Francisella tularensis subspecies holarctica from the country of Georgia. BMC Microbiol. 2011;11(1):139. http://dx.doi.org/10.1186/1471-218011-139 PMID:21682874

13. Gyuranecz M, Birdsell DN, Splettstoesser W, Seibold E, Beckstrom-Sternberg SM, Makrai L, et al. Phylogeography of Francisella tularensis subsp. holarctica, Europe. Emerg Infect Dis. 2012;18(2):290-3. http://dx.doi.org/10.3201/ eid1802.111305 PMID:22305204

14. Vogler AJ, Birdsell DN, Lee J, Vaissaire J, Doujet CL, Lapalus M, et al. Phylogeography of Francisella tularensis ssp. holarctica in France. Lett Appl Microbiol. 2011;52(2):177-80. http://dx.doi. org/10.1111/j.1472-765X.2010.02977.x PMID:21214606

15. Wang Y, Peng Y, Hai R, Xia L, Li H, Zhang Z, et al. Diversity of Francisella tularensis Subsp. holarctica Lineages, China. Emerg Infect Dis. 2014; 20:1191-4. http://dx.doi.org/10.3201/ eid2007.130931 PMID:24963721

16. Svensson K, Bäck E, Eliasson H, Berglund L, Granberg M, Karlsson L, et al. Landscape epidemiology of tularemia outbreaks in Sweden. Emerg Infect Dis. 2009;15(12):1937-47. http://dx.doi.org/10.3201/eid1512.090487 PMID:19961673

17. Larssen KW, Bergh K, Heier BT, Vold L, Afset JE. All-time high tularaemia incidence in Norway in 2011: report from the national surveillance. Eur J Clin Microbiol Infect Dis. 2014;33(11):1919-26. http://dx.doi.org/10.1007/s10096-0142163-2 PMID:24874046

18. Larssen KW, Afset JE, Heier BT, Krogh T, Handeland K, Vikøren $T$, et al. Outbreak of tularaemia in central Norway, January to March 2011. Euro Surveill. 2011;16(13):10-2. PMID:21489376

19. Harpest. [Tularaemia]. Solna: Folkhälsomyndigheten. [Accessed: 22 Dec 2014). Swedish. Available from: www.folkhalsomyndigheten.se/amnesomraden/ statistik-och-undersokningar/sjukdomsstatistik/harpest/

20. Simpson JT, Wong K, Jackman SD, Schein JE, Jones SJ, Birol I. ABySS: a parallel assembler for short read sequence data. Genome Res. 2009;19(6):1117-23. http://dx.doi.org/10.1101/ gr.089532.108 PMID:19251739

21. Langmead B, Salzberg SL. Fast gapped-read alignment with Bowtie 2. Nat Methods. 2012;9(4):357-9. http://dx.doi. org/10.1038/nmeth.1923 PMID:22388286

22. Koboldt DC, Zhang Q, Larson DE, Shen D, McLellan MD, Lin L, et al. VarScan 2: somatic mutation and copy number alteration discovery in cancer by exome sequencing. Genome Res. 2012;22(3):568-76. http://dx.doi.org/10.1101/gr.129684.111 PMID:22300766

23. Lärkeryd A, Myrtennäs K, Karlsson E, Dwibedi CK, Forsman M, Larsson P, et al. CanSNPer: a hierarchical genotype classifier of clonal pathogens. Bioinformatics. 2014;30(12):1762-4. http:// dx.doi.org/10.1093/bioinformatics/btu113 PMID:24574113

24. Svensson K, Sjödin A, Byström M, Granberg M, Brittnacher MJ, Rohmer L, et al. Genome sequence of Francisella tularensis subspecies holarctica strain FSC 200 , isolated from a child with tularemia. J Bacteriol. 2012;194(24):6965-6. http://dx.doi. org/10.1128/JB.01040-12 PMID:23209222

25. Darling AE, Mau B, Perna NT. progressiveMauve: multiple genome alignment with gene gain, loss and rearrangement. PLoS ONE. 2010;5(6):e11147. http://dx.doi.org/10.1371/journal. pone.0011147 PMID:20593022

26. Barabote RD, Xie G, Brettin TS, Hinrichs SH, Fey PD, Jay J, et al. Complete genome sequence of Francisella tularensis subspecies holarctica FTNFo02-00. PLoS ONE. 2009;4(9):e7041. http://dx.doi.org/10.1371/journal. pone.0007041 PMID:19756146

27. Petrosino JF, Xiang Q, Karpathy SE, Jiang H, Yerrapragada S, Liu Y, et al. Chromosome rearrangement and diversification of Francisella tularensis revealed by the type B (OSU18) genome sequence. J Bacteriol. 2006;188(19):6977-85. http://dx.doi. org/10.1128/JB.00506-06 PMID:16980500

28. Champion MD, Zeng Q, Nix EB, Nano FE, Keim P, Kodira CD, et al. Comparative genomic characterization of Francisella tularensis strains belonging to low and high virulence subspecies. PLoS Pathog. 2009;5(5):e1000459. http://dx.doi. org/10.1371/journal.ppat.1000459 PMID:19478886

29. Tamura K, Stecher G, Peterson D, Filipski A, Kumar S. MEGA6: Molecular Evolutionary Genetics Analysis version 6.0. Mol Biol Evol. 2013;30(12):2725-9. http://dx.doi.org/10.1093/molbev/ mst197 PMID:24132122

30. Johansson A, Lärkeryd A, Widerström M, Mörtberg S, Myrtännäs K, Öhrman C, et al. An outbreak of respiratory tularemia caused by diverse clones of Francisella tularensis. Clin Infect Dis. 2014;59(11):1546-53. http://dx.doi.org/10.1093/ cid/ciu621 PMID:25097081

31. Thelaus J, Andersson A, Broman T, Bäckman S, Granberg $M$, Karlsson L, et al. Francisella tularensis subspecies holarctica occurs in Swedish mosquitoes, persists through the developmental stages of laboratory-infected mosquitoes and is transmissible during blood feeding. Microb Ecol. 2014;67(1):96-107. http://dx.doi.org/10.1007/s00248-0130285-1 PMID:24057273

32. Padeshki PI, Ivanov IN, Popov B, Kantardjiev TV. The role of birds in dissemination of Francisella tularensis: first direct molecular evidence for bird-to-human transmission. Epidemiol Infect. 2010;138(3):376-9. http://dx.doi.org/10.1017/ S0950268809990513 PMID:19664305

33. Lopes de Carvalho I, Zé-Zé L, Alves AS, Pardal S, Lopes RJ, Mendes $\mathrm{L}$, et al. Borrelia garinii and Francisella tularensis subsp. holarctica detected in migratory shorebirds in Portugal. Eur J Wildl Res. 2012;58(5):857-61. http://dx.doi.org/10.1007/ S10344-012-0617-3

34. Gürcan S, Karabay O, Karadenizli A, Karagöl C, Kantardjiev T, Ivanov IN. Characteristics of the Turkish isolates of Francisella tularensis. Jpn J Infect Dis. 2008;61(3):223-5. PMID:18503176 